\title{
A note on "On fast trust region methods for quadratic models with linear constraints", by Michael J.D. Powell
}

\author{
Daniel Bienstock ${ }^{1}$ • Philip E. Gill ${ }^{2}$ • Nick Gould ${ }^{3}$
}

Published online: 29 May 2015

(C) Springer-Verlag Berlin Heidelberg and The Mathematical Programming Society 2015

The following paper, "On fast trust region methods for quadratic models with linear constraints" by Michael J.D. Powell, was submitted to Mathematical Programming Computation in August 2014, and was still in review when we became aware of Mike's deteriorating health. Unfortunately, despite our best efforts and those of two referees, Mike died before we were able to send him our verdict. Both referees had concerns about points that needed further explanation, but in all likelihood-and after a few clarifying iterations - the paper would ultimately have been published. As we believe that the ideas central to the paper are of general interest to the journal's readership, we have taken the unusual step of publishing the paper "as is". Readers might, like the referees, question some of the statements made, but we feel it would be wrong to second guess Mike's intentions, and have chosen to leave such statements alone.

Mike had a profound effect on computational nonlinear optimization. We dedicate this paper to his memory.

We express our thanks to Andy Conn and another referee for dropping everything to get their reports to us, and for accepting that publication was the best option. We also thank Arieh Iserles for locating the LaTeX version of the manuscript amongst Mike Powell's files.

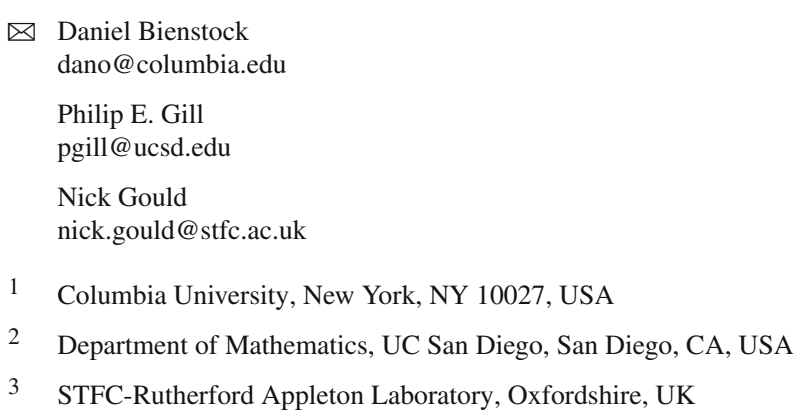

